\title{
Recubrimientos de hidroxiapatita preparados mediante un proceso sol-gel
}

\author{
E. Peón ${ }^{* * *}$, A. Jiménez-Morales ${ }^{* * *}$, E. Fernández-Escalante*, M.C. García-Alonso*, \\ M.L. Escudero* y J.C. Galván*
}

Resumen Se han preparado nuevos recubrimientos de hidroxiapatita (HAp) obtenidos a partir de precursores de tipo sol-gel, depositados sobre sustratos metálicos de una aleación de base hierro denominada MA956, previamente oxidados a elevadas temperaturas, para mejorar la adherencia con los recubrimientos de hidroxiapatita. El recubrimiento sol-gel se obtuvo aplicando una ruta acuosa, utilizando como precursores del fósforo y del calcio trietilfosfito y nitrato cálcico tetrahidratado, respectivamente. Sobre los geles resultantes se aplicaron distintos tratamientos térmicos de sinterización, hasta la obtención de recubrimientos de hidroxiapatita homogéneos, adherentes y cristalinos. Las técnicas de caracterización utilizadas para realizar este estudio han sido microscopía óptica, MBE/EDX, DRX y FTIR. Así mismo, se ha determinado la adherencia entre el substrato y el recubrimiento de hidroxiapatita según la norma ASTM D 3359-02. Los resultados obtenidos han mostrado que el tratamiento térmico óptimo se consigue cuando se aplica una temperatura de sinterización de $550^{\circ} \mathrm{C}$ durante un tiempo igual a $72 \mathrm{~h}$.

Palabras clave Hidroxiapatita. Recubrimientos sol-gel. Biomateriales. Aleación MA956.

\section{Hydroxyapatite coatings prepared by a sol-gel process}

\begin{abstract}
Hydroxyapatite coatings (HAp) have been obtained starting from precursors of sol-gel type. The coatings previously oxidized were deposited on metal surfaces of a based on iron alloy so-called MA956. The alloys were at high temperatures, in order to improve the adhesion with the hydroxyapatite coatings. The sol-gel coating was obtained applying an aqueous route, using triethyl phosphite and aqueous calcium nitrate, as precursors of phosphorous and calcium, respectively. Different sintering thermal treatments were applied on the resulting gels in order to obtain a homogeneous, adherent and crystalline hydroxyapatite coating. The characterization techniques used for this study were optical microscopy, SEM/EDX, XDR and FTIR. Moreover, the adhesion between the hydroxylapatite coating and the substrate was assessed according to the ASTM D 3359-02 standard test method. The results of this study showed that the best thermal treatment is obtained for a sintering temperature of $550^{\circ} \mathrm{C}$ during a time of $72 \mathrm{~h}$.
\end{abstract}

Keywords Hydroxyapatite. Sol-gel coatings. Biomaterials. MA956 alloy.

\section{INTRODUCCIÓN}

En la década del 80 comienzan a desarrollarse los recubrimientos de hidroxiapatita sobre sustratos metálicos y pronto comienza a comercializarse este tipo de recubrimientos, para aplicaciones en el campo de los biomateriales. Sin embargo, todavía hoy en día, persisten problemas relacionados con la uniformidad del recubrimiento y la estabilidad a largo plazo de la unión del recubrimiento al sustrato metálico. Actualmente, se realizan innumerables investigaciones para desarrollar un sistema que combine las ventajas mecánicas de los metales y la afinidad biológica de las cerámicas de hidroxiapatita, obtenidos por diferentes vías y depositados sobre superficies metálicas, tratando de superar los inconvenientes arriba mencionados ${ }^{[1}$ y 2$]$. En este contexto, se están aplicando los procesos sol-gel

(*) Centro Nacional de Investigaciones Metalúrgicas (CENIM-CSIC), Avda. Gregorio del Amo, 8, 28040 Madrid, España.

$(* *) \quad$ Centro de Biomateriales, Universidad de La Habana, C. Habana 10600, Cuba.

(***) Universidad Carlos III de Madrid, Avda. Universidad 30, Leganés, España. 
que ofrecen importantes ventajas sobre otras técnicas de recubrimiento. Gracias a la aplicación de esta tecnología, se consiguen recubrimientos con una mayor homogeneidad debido a que los procesos de mezcla ocurren a escala nanoscópica, reduciéndose además la temperatura de sinterización, debido al pequeño tamaño de partícula. Con esta técnica se logra recubrir, fácilmente, formas complejas, pudiéndose emplear, asimismo, diversas técnicas de deposito para la producción de dichos recubrimientos (rociado, inmersión, aplicación centrífuga, etc.). Además, gracias a la versatilidad de los procesos sol-gel se pueden optimizar las condiciones de síntesis empleadas, de tal forma que los materiales resultantes presenten las propiedades específicas deseadas, que pueden ser elegidas de maneras tan dispares como para que el resultado final sea desde un material de elevada superficie específica y porosidad, hasta un material denso ${ }^{[2}$ y 3$]$. Dentro de este contexto, en la presente comunicación se muestran resultados preliminares de un estudio integrado en el que se contemplan aspectos tan diversos como son la preparación, el diseño y la caracterización de recubrimientos de hidroxiapatita sobre aleaciones de base hierro o titanio, para posibles aplicaciones biomédicas.

\section{MATERIALES Y PROCEDIMIENTO EXPERIMEN- TAL}

Como sustratos metálicos se ha utilizado una superaleación denominada MA956, de composición nominal Fe-20Cr-5Al-0,5Ti-0,5Y2O3. Sobre la superaleación MA956 se aplicó previamente un tratamiento térmico a $1.100{ }^{\circ} \mathrm{C}$ durante $8 \mathrm{~h}$, para producir una película homogénea y compacta de $\alpha$-alumina que previsiblemente, actuará, al mismo tiempo, como barrera frente a la corrosión del sustrato metálico ${ }^{[4]}$ y como promotor de la adherencia con el recubrimiento de hidroxiapatita.

El recubrimiento de hidroxiapatita cristalina, se ha preparado aplicando una variante del proceso sol-gel descrito por D. M. Liu ${ }^{\left[3 y^{5]}\right.}$. Los soles se han obtenido utilizando como reactivos trietil fosfito y nitrato cálcico tetrahidratado. La hidrólisis del precursor del fósforo (solución alcohólica de trietil fosfito) se provoca bajo agitación vigorosa en un medio acuoso, utilizando una relación molar agua/fosfito igual a 4 . Tras 24 h de hidrólisis, se añade al medio una cantidad estequiométrica de solución alcohólica $3 \mathrm{M}$ de nitrato del calcio, manteniéndolo bajo agitación durante $3 \mathrm{~min}$, dejándolo a continuación en reposo, a temperatura am- biente, otras $24 \mathrm{~h}$, para obtener el gel de hidroxiapatita. Cuando el gel alcanza la viscosidad adecuada, se deposita sobre la superficie metálica mediante inmersión, a una velocidad de $5 \mathrm{~cm} / \mathrm{min}$. Por último, se procede a la sinterización del recubrimiento de hidroxiapatita aplicando diferentes tratamientos térmicos hasta optimizar la temperatura y el tiempo de tratamiento más adecuados. En este contexto, se ha aplicado un novedoso método de tratamiento térmico, consistente en una variante de la técnica de análisis térmico a velocidad controlada (ATVC) de Rouquerol ${ }^{[6 \text { y } 7]}$ con el objeto de que, además de la cristalización y densificación del recubrimiento de hidroxiapatita, se controlen también sus características de textura. El recubrimiento resultante de hidroxiapatita se ha caracterizado mediante espectroscopía infrarroja (FTIR), difracción de rayos X (DRX), microscopía electrónica de barrido (MEB/EDX). Asímismo, se ha determinado la adherencia entre el sustrato y el recubrimiento de hidroxiapatita, siguiendo las especificaciones de la norma ASTM D 3359-02 ${ }^{[8]}$.

\section{RESULTADOS Y DISCUSIÓN}

Mediante microscopía óptica se estudió la morfología de las capas de hidroxiapatita formadas sobre el sustrato metálico, a las diferentes temperaturas de tratamiento térmico de sinterización aplicado. Así, se comprobó que por debajo de $350^{\circ} \mathrm{C}$ no se produce una completa cristalización de la hidroxiapatita y que las capas depositadas, todavía, no son homogéneas. Al alcanzar dicha temperatura se observa el comienzo del crecimiento de pequeños cristales de hidroxiapatita a partir de múltiples centros de nucleación (Fig. 1a). El aumento progresivo de la temperatura muestra que la cristalización completa de la hidroxiapatita se logra al alcanzar una temperatura de tratamiento de $550^{\circ} \mathrm{C}$ (Fig. 1b).

Con los estudios de microscopía electrónica de barrido (MEB) se observó que el recubrimiento resultante presenta una pequeña porosidad con una morfología homogénea y sin grietas (Fig. 2).

El análisis mediante energía dispersiva de rayos $\mathrm{X}(\mathrm{EDX})$ mostró que el calcio y el fósforo están presentes en el recubrimiento de hidroxiapatita en una relación molar $\mathrm{Ca} / \mathrm{P}$ de 1,66 (Fig. 3), sin que hayan aparecido picos característicos de los distintos sustratos metálicos, evidenciándose la continuidad de la capa de hidroxiapatita sobre el sustrato metálico. 
Recubrimientos de hidroxiapatita preparados mediante un proceso sol-gel E. Peón, A. Jiménez-Morales, E. Fernández-Escalante, M.C. García-Alonso, M.L. Escudero y J.C. Galván
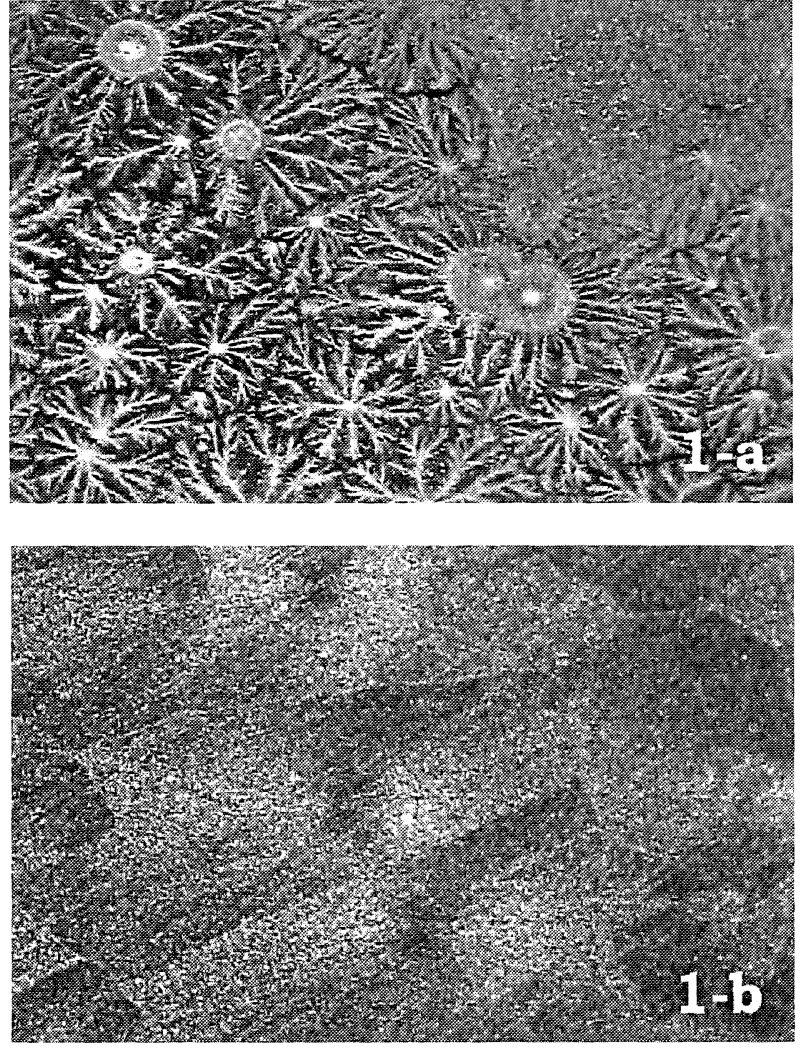

Figura 1. Morfología de las capas depositadas de HAp sobre la aleación MA956, tras aplicar tratamientos térmicos a diferentes temperaturas: a) $350^{\circ} \mathrm{C}$ y b) $550^{\circ} \mathrm{C}$.

Figure 1. Morphology of the HAp layers deposited on the MA956 alloy, after applying thermal treatments at different temperatures: a) $350^{\circ} \mathrm{C}$ and, b) $550^{\circ} \mathrm{C}$.

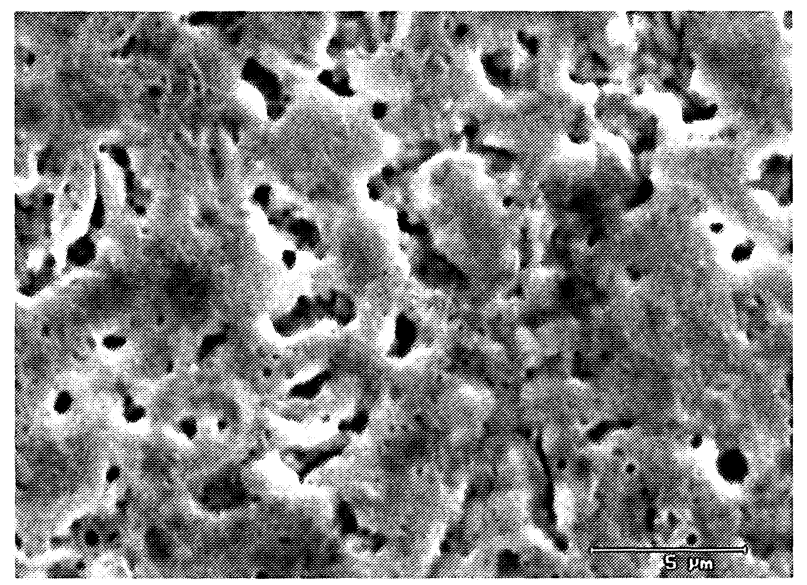

Figura 2. Micrografía MEB de un recubrimiento de HAp, obtenido a partir de trietil fosfito y nitrato cálcico, depositado sobre un sustrato metálico preoxidado de MA956 y sinterizado a $550^{\circ} \mathrm{C}$.

Figure 2. SEM micrography of a HAp coating obtained from triethyl phosphite and calcium nitrate, deposited on the MA956 preoxidized alloy and sintered at $550^{\circ} \mathrm{C}$.

La figura 4 muestra un ejemplo representativo de los difractogramas obtenidos, donde las líneas

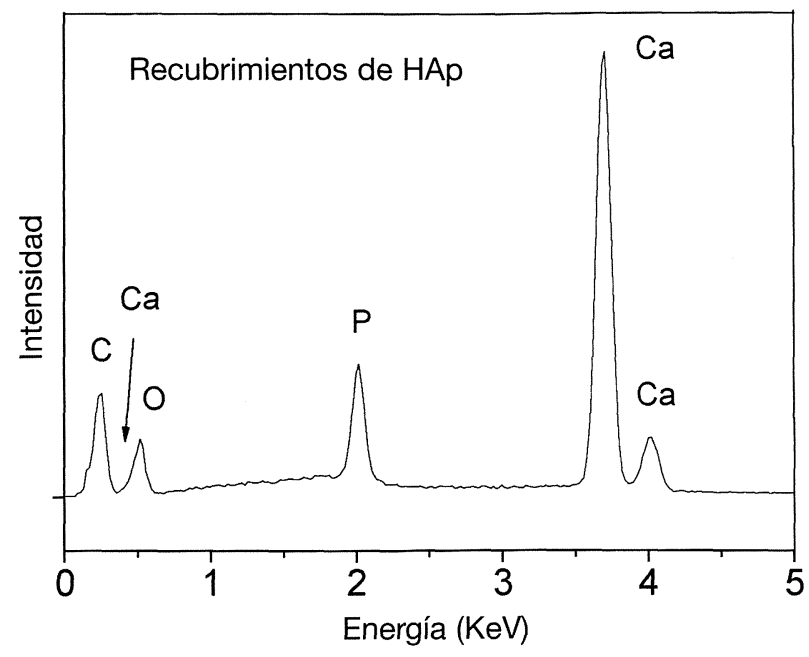

Figura 3. Análisis EDX de un recubrimiento de HAp, obtenido a partir de trietil fosfito y nitrato cálcico, depositado sobre un sustrato metálico de MA956 preoxidado.

Figure 3. EDX analysis of a HAp obtained from triethyl phosphite and calcium nitrate, deposited on the MA956 preoxidized alloy.

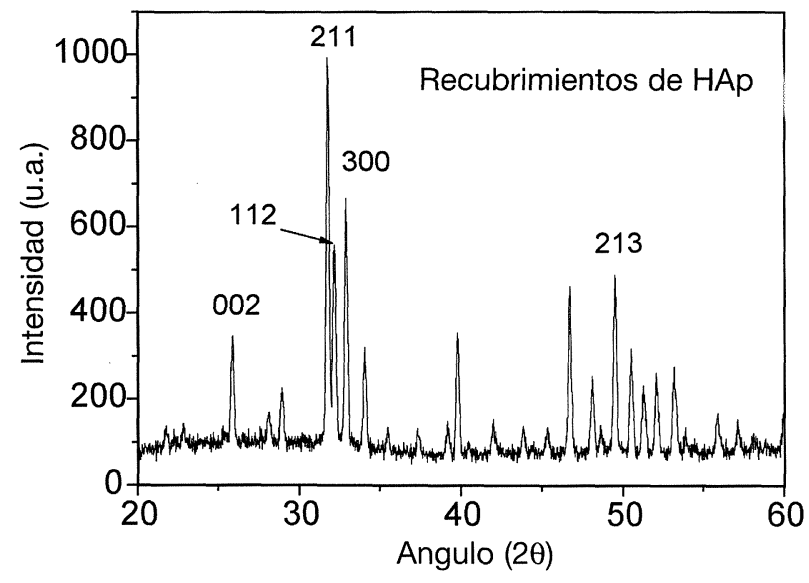

Figura 4. Difractograma de un recubrimiento de HAp, obtenido a partir de trietil fosfito y nitrato cálcico, depositado sobre la aleación MA956 preoxidada.

Figure 4. XDR pattern of a HAp coating obtained triethyl phosphite and calcium nitrate, deposited on the MA956 preoxidized alloy.

características para este material corresponden a una única fase cristalina identificada como hidroxiapatita. En todos los casos, se obtuvieron difractogramas similares, donde las líneas que se observan son idénticas a las reportadas en la literatura para la hidroxiapatita ${ }^{[9]}$. Los picos observados a $25,88^{\circ}(002), 31,76^{\circ}$ (211), el doblete de $32,18^{\circ}$ (112) y $32,90^{\circ}(300)$, así como los que aparecen a $49,50^{\circ}$ (213), característicos de la HAp, sugieren que las capas de fosfato de calcio evolucionaron de un estado amorfo a cristalino. Como se puede 
apreciar no existen picos correspondientes a otras fases de fosfatos de calcio que no se correspondan con la HAp estequiométrica y bien cristalizada.

La figura 5 muestra el espectro IR (región 400. $1.300 \mathrm{~cm}^{-1}$ ) de las capas de hidroxiapatita tratadas a $550^{\circ} \mathrm{C}$. Se observan las bandas características de las vibraciones asociadas a ${ }_{4} \mathrm{PO}_{4}^{3-}$ a los 563 y 600 $\mathrm{cm}^{-1}, \mathrm{v}_{1} \mathrm{PO}_{4}^{3-}$ a los $942 \mathrm{~cm}^{-1}, \mathrm{y} \mathrm{v}_{3} \mathrm{PO}_{4}{ }^{3-}$ a los 1.100 y $1.000 \mathrm{~cm}^{-1}$, respectivamente. Estas bandas indican el ordenamiento de los poliedros de $\mathrm{PO}_{4}{ }^{3-}$ en la estructura del cristal. La banda que aparece a $631 \mathrm{~cm}^{-1}$ se atribuye a los grupos $\mathrm{OH}^{-}$. Tanto los resultados de espectroscopia infrarroja (FTIR) como los de difracción de rayos $\mathrm{X}$ muestran la cristalinidad de la fase de hidroxiapatita formada.

La adherencia entre el sustrato y el recubrimiento de hidroxiapatita se ha determinado aplicando los ensayos recomendados en la norma ASTM D 3359-02 ${ }^{[8]}$. A pesar de tener un carácter cualitativo, dichos ensayos representan una aproximación muy útil para evaluar las propiedades de adherencia, por lo que no es de extrañar que se apliquen habitualmente en estudios de recubrimientos orgánicos e, incluso, de recubrimientos de tipo sol-gel ${ }^{[10-12]}$. Los recubrimientos que presentaron la mejor adherencia aplicando estos ensayos son los que se trataron a una temperatura de sinterización de $550^{\circ} \mathrm{C}$ durante $72 \mathrm{~h}$. Los resultados obtenidos mostraron que estos recubrimientos presentan un grado de adherencia igual a 5B, antes de aplicar la cinta adhesiva, el cual en ocasiones disminuye al grado 4B, después de aplicar la cinta adhesiva.

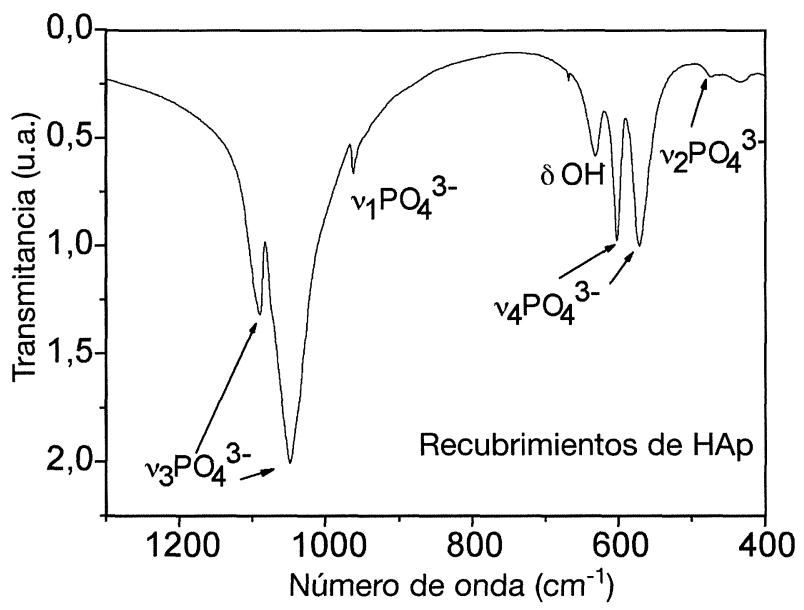

Figura 5. Espectro IR del recubrimiento de HAp, obtenido a partir de trietil fosfito y nitrato cálcico, depositado sobre la aleación MA956 preoxidada.

Figure 5. IR spectrum of a HAp coating obtained from triethyl phosphite and calcium nitrate, deposited on the MA956 preoxidized alloy.

\section{CONCLUSIONES}

Se han obtenido recubrimientos de hidroxiapatita sobre superficies metálicas preoxidadas, a partir de precursores de tipo sol-gel preparados con trietil fosfito y nitrato cálcico tetrahidratado. Los tratamientos térmicos de sinterización aplicados sobre el recubrimiento sol-gel muestran que, para un tiempo de sinterización de $72 \mathrm{~h}$, la temperatura de tratamiento mas adecuada es $550^{\circ} \mathrm{C}$. Con este tratamiento se consigue un recubrimiento cristalino, homogéneo y fuertemente adherido al sustrato metálico.

\section{Agradecimientos}

El presente trabajo ha sido financiado por un proyecto de investigación de la Comunidad de Madrid (Ref. 07N/0082/2002) y por un proyecto conjunto CSIC/CITMA-Cuba (Ref. 2001CU0004).

\section{REFERENCIAS}

[1] I.S. LeE, H.E. Kim, S.Y. KIM, Surf. Coat. Technol. 131 (2000) 181-186.

[2] Q.L. Feng., F.Z. Qui, H. WanG, T.N. Kim, J.O. KIM, J. Crystal Growth 210 (2000) 735-740.

[3] D.M. LiU, T. Troczynski, W.J. TsenG; Biomaterials 22 (2001) 1.721-1.730.

[4] M.C. García-Alonso, J.L. González-Carrasco, P. Pérez, V.A.C. HaAnappel, M.L. Escudero, J. Chao, M.F. StroosnijDer, J. Mater. Sci.: Mater. Med. 12 (2001) 589-596.

[5] D.M. LiU, Q. YANG, T. TroczynsKI, Biomaterials 23 (2002) 691-698.

[6] J.C. Galván, A. Jiménez-Morales, R. Jiménez, A. Villanueva, M. Crespin, P. Aranda, E. Ruiz-Hitzky, Chem. Mater. 10 (1998) 3.379-3.385.

[7] a) J. ROUQUeROL, Termochim. Acta 144 (1989) 209-244. b) S. Bordère, F. RouQuerol, J. Estienne y A. Floreancig, J. Therm. Anal. Calorim. 36 (1990) 1.6511.668.

[8] ASTM D 3359-02, Standard Test Methods for Measuring Adhesion by Tape Test, ASTM International.

[9] Joint Committee for Powder Diffraction Standards (JCPDS Card No. 9-432).

[10] C. Schelle, M. Mennig, H. Krug, G. Jonschker, H. SCHMIDT, J. Non-Crystalline Solids 218 (1997) 163-168.

[11] R.A. Sailer, M.D. SouceK, Progress Org. Coat. 33 (1) (1998) 36-43.

[12] D. Deffar, G. Teng, M.D. Soucek, Surf. Coat. Int. Part B-Coatings Trans. 84 (2) (2001) 147-156.

Rev. Metal. Madrid Vol. Extr. (2005) 479-482 